\title{
Plasma Immersion Ion Implantation of Stainless Steel 316
}

\author{
J. Davis*, K. Short*, R. Wuhrer**, M. R. Phillips** and K. Whittle* \\ * Institute of Materials Engineering, Australian Nuclear Science and Technology Organization. \\ ** Microstructural Analysis Unit, Faculty of Science, University of Technology, Sydney.
}

Plasma immersion ion implantation $\left(\mathrm{PI}^{3}\right)$ is a method designed to implant large areas of samples simultaneously, to very high fluences, with a small energy variation. It originally grew out of an ANSTO project in the 1980's investigating the fundamental properties of plasmas relevant for use in nuclear fusion. The process involves forming an ionised plasma at a controlled temperature with radio frequency (RF) excitation. Ion implantation occurs when the positive ions from the plasma are accelerated towards all exposed surfaces by applying a high negative voltage to the sample. It has previously been used for surface hardening and improving corrosion properties of metals. This ion implantation technique is being used to simulate the effects from a neutron irradiation environment and study the effects on various materials.

Research is currently in progress to determine the effect of $\mathrm{PI}^{3}$ has on a 316 stainless steel microstructure, utilising argon and helium ions as damaging ions. The overall aim of the research is to eventually investigate other unique materials that have applications in both GenIV and fusion reactor programs, and the Advanced Fuel Cycles (AFCI) e.g. inert matrix fuels, reactor core liners, structural materials within the reactor (ODS materials). The main focus of this work will be to understand grain-boundary interactions, and how they relate to the tolerance of materials to damage.

A small piece of 316 stainless steel was prepared for EBSD analysis using the Struers method for polishing Low Carbon Steels (Method No. 1879). The sample was then mapped using a Zeiss Ultra Plus SEM with an Oxford Instruments HKL NordlysS detector system. The map was generated using the HKL Channel 5 software. Ion implantation utilising two different noble gases, argon and helium respectively at $40 \mathrm{kV}$ accelerating voltage, and to a fluence of $\sim 1 \times 10^{17}$ ions $\mathrm{cm}^{-2}$ was used. Portions of the materials were shielded using both a silicon wafer and a TEM grid. Post irradiation EBSD was used to analyse both the shielded and non-shielded surfaces.

Fig. 1 shows the BSE image of the irradiated and shielded regions and also the region where the EBSD map was taken from on the polished stainless steel surface, where a) Argon ions were used for implantation and b) Helium ions were used for implantation. Fig. 2a shows the EBSD map of the type 316 stainless steel before ion implantation and Figure $2 \mathrm{~b}$ shows the ccorresponding EBSD map showing the loss of EBSD pattern in the irradiated region.

$\mathrm{PI}^{3}$ using argon and helium ions significantly degraded the backscattered electron image and EBSD patterns on the 316 stainless steel sample. Irradiation induced dislocations/damage affected the crystallinity of the surface. Physical damage from the irradiation to the surface preparation for EBSD, removing the coherent scattering, and effectively gave rise to diffuse scattering from the surface.

Current research and future research investigation of these and many other materials will be discussed. Cross sections of the irradiated steel are being prepared to determine the penetration depth of the damage and whether EBSD patterns are obtainable from the cross section. The affect of type of ion will also be discussed. Helium ions should have penetrated further than argon, predominantly due to the mass/energy ratio of argon/helium. Further experiments will be focused 
on a range of doses in combination with nano-indentation, to study the effect of radiation on the recovery from stress in materials, and the role it plays in recovery.

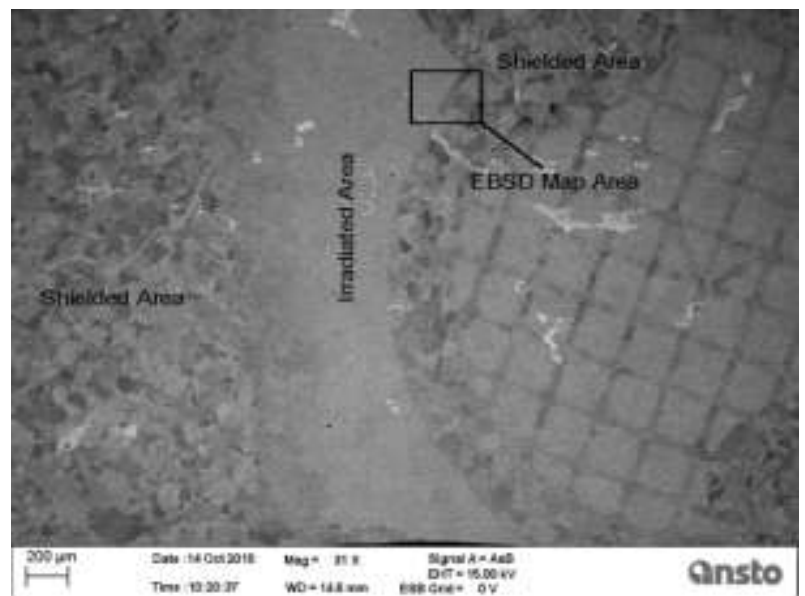

a.

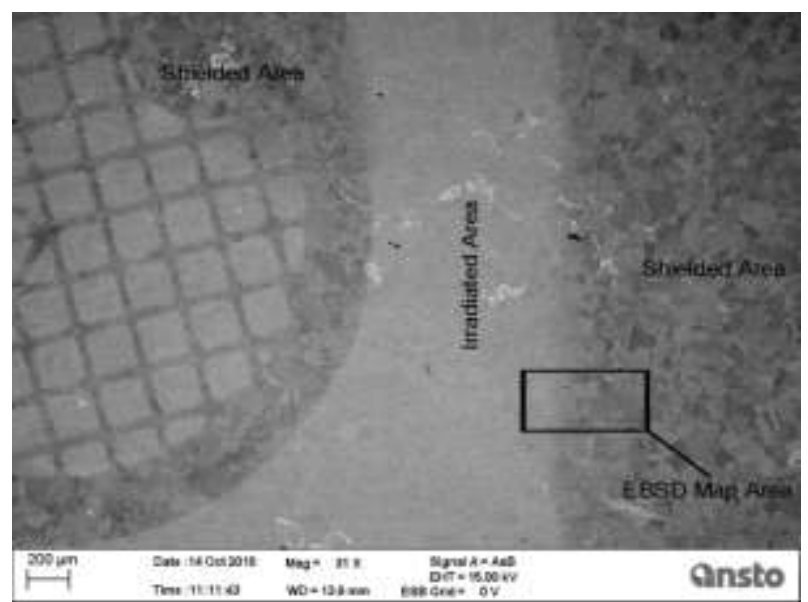

b.

Figure 1. Backscattered electron image showing the irradiated and shielded regions and also the region where the EBSD map was taken from on the polished stainless steel surface. a) Argon ions were used for implantation and b) Helium ions were used for implantation.

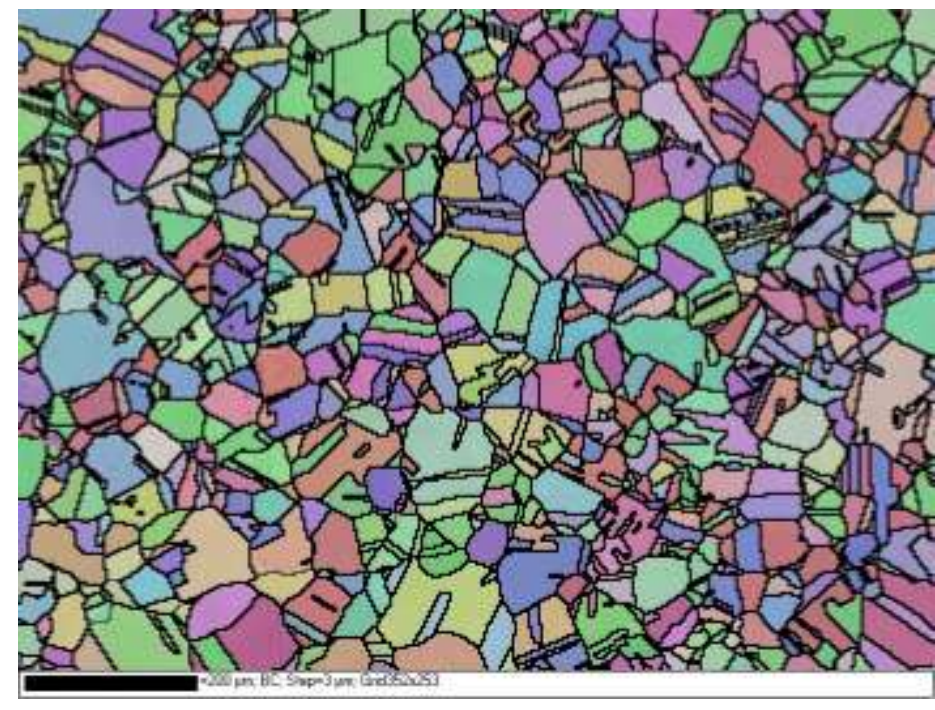

a.

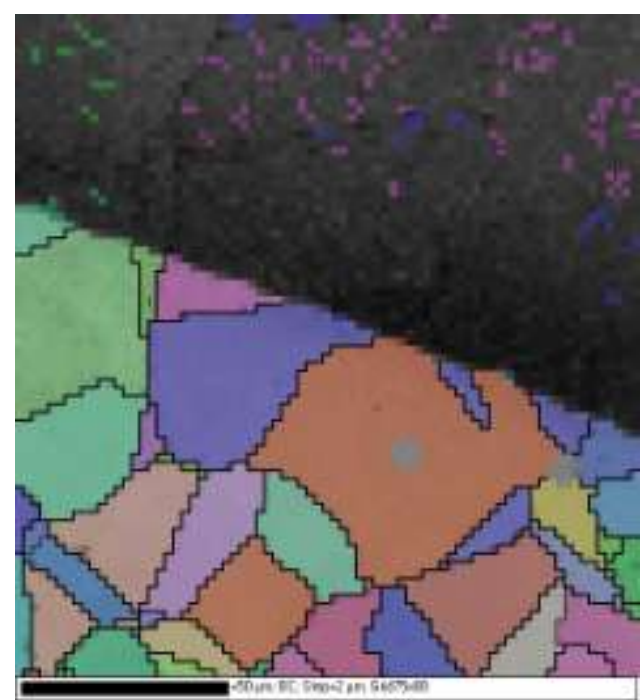

b.

Fig. 2. EBSD map showing the loss of EBSD pattern in the irradiated region (top of map). 